\title{
CORRELATED O AND Mg ISOTOPIC ANOMALIES IN ALLENDE INCLUSIONS: II. MAGNESIUM
}

\author{
G. J. Wasserburg, Typhoon Lee and D. A. Papanastassiou \\ The Lunatic Asylum, Div. of Geological and Planetary Sciences, California Institute of Technology, Pasadena, California 91125
}

\begin{abstract}
Mg}$ in two Allende $\mathrm{Ca}-\mathrm{Al}$ rich inclusions shows large isotopic, mass-dependent fractionation which enriched the hebvier isotopes. After normalization, $\mathrm{Mg}$ in these inclusions shows negative $\delta^{26} \mathrm{Mg}$ which appears to require the presence of nuclear effects in $\mathrm{Mg}$ distinct from ${ }^{26} \mathrm{Al}$ decay. The $\mathrm{Mg}$ mass fractionation is correlated with distinct but smaller fractionation effects for $\mathrm{O}$ reported by Clayton and Mayeda for the same inclusions (see companion paper). The observation of distinctive but uniform $\mathrm{Mg}$ isotopic composition in different phases within single Allende inclusions indicates that nuclear effects in $\mathrm{O}$ and $\mathrm{Mg}$ are not due to the entrapment of interstellar carrier grains as discrete entities, which are preserved as remnants, but are instead due to a homogenized mixture of components of extraordinary isotopic composition mixed with a component of ordinary solar system material and subjected to isotopic fractionation. The distinct $O$ isotopic composition of different phases within a single inclusion is believed to be due to incomplete back-reaction of the higher temperature condensates with a cooler solar nebula of "normal" composition. The processes responsible for the $\mathrm{O}$ and $\mathrm{Mg}$ nuclear effects and the astrophysical site of their occurrence remain undefined.
\end{abstract}

This note presents the observation in three Allende inclusions of large isotopic fractionation effects in $\mathrm{Mg}$; two of the samples show a correlation between $\mathrm{Mg}$ and $\mathrm{O}$ isotopic anomalies and indicate possible nuclear effects in $\mathrm{Mg}$. In general, the effect of small fractionation on the isotopic ratios of an element is given approximately by

$$
\mathbf{R}_{1 \mathrm{j}} / \mathbf{R}_{\mathbf{1 j}}^{0}=1+\alpha\left(\mathbf{M}_{1}-\mathbf{M}_{\mathrm{j}}\right)
$$

where $R_{1 j}$ is the measured ratio of the isotopes of mass $M_{1}$ and $M_{j}, R_{1 j}^{0}$ is the "normal unfractionated" ratio, and $\alpha$ is the fractionation factor per unit of mass difference which includes the fractionation intrinsic in the sample and that produced by the analytical procedures. A key issue is the ability to distinguish between fractionation in the sample and that produced by analytical procedures. We define any variation in isotopic ratios which does not satisfy equation (1) as a non-linear isotopic effect. Catanzaro and Murphy [1966] showed that the $\mathrm{Mg}$ fractionation is less than $1 \%$ oer mu (mass unit) in terrestrial samples. In a search for effects due to ${ }^{26} \mathrm{Al}$, Schramm, Tera and Wasserburg [STW, 1970] studied $\mathrm{Mg}$ in a variety of samples using a technique with high precision and sensitivity. These workers normalized their data to a standard value of ${ }^{25} \mathrm{Mg} /{ }^{24} \mathrm{Mg}$ to determine $\alpha$ and corrected ${ }^{26} \mathrm{Mg} /{ }^{24} \mathrm{Mg}$ for fractionation using eq. (1) to obtain deviations in ${ }^{26} \mathrm{Mg} /{ }^{24} \mathrm{Mg}$ in permil relative to the standard. These arbitrarily normalized deviations are defined as $\delta^{26} \mathrm{Mg}$. $S T W$ showed that there were no variations in $\delta^{26} \mathrm{Mg}$ within $\pm 0.2 \%$ and thereby demonstrated the absence of any non-linear effects in the samples investigated. In 1974 two independent groups [Gray and Compston, 1974; Lee and Papanastassiou ( $L \& P)$, 1974] reported non-linear isotopic effects in $\mathrm{Mg}$ extracted from inclusions in the Allende meteorite. These inclusions had previously been shown to be chemically similar to early condensates from the solar nebula [Lord, 1965; Grossman, 1972] and to contain the most primitive $\mathrm{Sr}$ so far observed [Gray, Papanastassiou and Wasserburg, 1973]. This type of inclusions had also been shown by Clayton, Grossman and Mayeda [1973] to contain an extraordinary oxygen isotopic component whose presence was attributed by them to the entrapment of interstellar dust grains. Lee, Papanastassiou and Wasserburg $(L P W)[1976,1977]$ established that their instru-

Copyright 1977 by the American Geophysical Union. mental fractionation has a well-defined range of $\pm 3 \%$ per mu and showed that there existed samples with normal ${ }^{25} \mathrm{Mg} /{ }^{24} \mathrm{Mg}$ but large well-resolved positive non-linear anomalies in ${ }^{26} \mathrm{Mg} /{ }^{24} \mathrm{Mg}$ of up to $10 \%$ which correlate with $\mathrm{Al} / \mathrm{Mg}$ for well-defined mineral phases within individual inclusions. These data demonstrated the existence of ${ }^{26} \mathrm{Al}$ in the early solar system. The work of $L \& P$ [1974] showed no correlation for "whole" inclusions between the $\mathrm{Mg}$ and $\mathrm{O}$ anomalies. In addition, whereas spinel shows the largest $O$ anomaly among phases in Allende [Clayton et al., 1977], normal Mg was found in spinels. $L \& P$ [1974] found one sample (C1) with a negative $\delta^{26} \mathrm{Mg}\left(\delta^{24} \mathrm{Mg}\right)$ of $-1.7 \%$ and with raw ${ }^{25} \mathrm{Mg} /{ }^{24} \mathrm{Mg}$ and ${ }^{26} \mathrm{Mg} /{ }^{24} \mathrm{Mg} \sim 30 \%$ ond $\sim 58 \%$ o higher than "normal", although it was not evident whether these large effects represented natural or instrumental fractionation. Heymann and Dziczkaniec [1976] suggested that this negative anomaly could be an important clue to nuclear processes in the early solar system and presented a model to explain the negative $\delta^{26} \mathrm{Mg}$.

A series of investigations undertaken by $L P W$ [1976] showed that the apparent large mass fractionation and the negative $\delta^{26} \mathrm{Mg}$ anomaly were real and that different phases in Cl have the same $\mathrm{Mg}$ isotopic composition which is fractionated with a marked enrichment of ${ }^{26} \mathrm{Mg}$ relative to ${ }^{25} \mathrm{Mg}$ and ${ }^{25} \mathrm{Mg}$ relative to ${ }^{24} \mathrm{Mg}$. This demonstrated that isotopic fractionation processes were active prior to or during the formation of some inclusions. Sample $\mathrm{Cl}$ was sent to R.N. Clayton for oxygen analysis and was found to lie distinctly off the mixing line between "normal" solar system oxygen $(N)$ and an extraordinary component (E) consisting of pure ${ }^{16} \mathrm{O}$ [Clayton et al., 1977]. Thus this unique sample was the only one which hinted at a relationship between $\mathrm{O}$ and $\mathrm{Mg}$ anomalies. Clayton and Mayeda $[1977 \mathrm{a}, \mathrm{b}]$ reported data on a second Ca-Al inclusion (EK 1-4-1) which fall off the NE mixing line for many inclusions in Allende. Dr. Nagasawa was kind enough to supply us with material at the request of $R$. Clayton.

Experimental Results. Samples of EK 1-4-1 included: A) 4 $\mathrm{mg}$ of a density separate consisting of pyroxene with abundant spinel inclusions; and B) $4 \mathrm{mg}$ of spinel obtained by $\mathrm{HF}$ treatment of separate A. Spinel crystals from B were analyzed using the direct loading technique [Wasserburg et al., 1977]. An aliquot of $\mathrm{A}$ was dissolved in $\mathrm{HF}$ and centrifuged. The soluble part was subject to our standard separation procedures and analyzed. The insoluble residue, consisting almost exclusively of spinel, was fused and the $\mathrm{Mg}$ was chemically separated and analyzed. Electron microprobe analyses on grains from $B$ and the residue of A showed that they were all spinel. A survey of twenty-six grains from A, excluding spinel inclusions, showed that they were all $\mathrm{Ca}-\mathrm{Al}$ rich pyroxene with variable $\mathrm{Al}$ and $\mathrm{Ti}$ contents $\left(<0.1\right.$ to $\left.6.6 \% \mathrm{TiO}_{2}\right)$. New data and the results for $\mathrm{C} 1$ are shown in Tables 1\&2. Raw data for each experiment are shown in Fig. 1. Histograms of the raw data for all normal samples analyzed over the past four years are shown. Data on ${ }^{25} \mathrm{Mg} /{ }^{24} \mathrm{Mg}$ for the vast majority of Allende samples would also plot within the distribution of normals. All data on normals show that the extent of instrumental fractionation is well defined; the observed range (99.5\% of the data) is $\pm 3 \%$ or for ${ }^{25} \mathrm{Mg} /{ }^{24} \mathrm{Mg}$ and $\pm 6 \%$ for ${ }^{26} \mathrm{Mg} /{ }^{24} \mathrm{Mg}$. All EK1-4-1 samples showed large fractionation effects of $\alpha \approx 20 \%$ per mu which are far outside of the range for normal samples. In addition the normalized data give $\delta^{26} \mathrm{Mg} \cong-3.7 \pm 0.3 \%$ for spinel and fassaite from A. A review of our earlier data on Allende showed other specimens which indicated fractionation. A fine-grained aggregate B29 which showed a positive $\delta^{26} \mathrm{Mg}[L \& P, 1974]$ was of interest as the 
Table 1. Isotopic Results of FUN Samples

\begin{tabular}{|c|c|c|c|c|c|c|c|c|c|c|}
\hline \multirow[t]{3}{*}{ Sample } & \multirow[t]{3}{*}{ Sets $^{a}$} & \multicolumn{6}{|c|}{ Unnormalized Ratios $^{b}$} & \multirow{3}{*}{$\begin{array}{l}\text { Normalized } \\
\delta^{26} \mathrm{Mg}(\% \circ)\end{array}$} & \multirow{3}{*}{$\begin{array}{l}\delta^{17} 0 \\
(\% \text { o }) \\
\text { SMOW }\end{array}$} & \multirow{3}{*}{$\begin{array}{l}\delta^{18} \mathrm{O} \\
(\% \circ) \\
\text { SMOW }\end{array}$} \\
\hline & & & ${ }^{25} \mathrm{Mg} /{ }^{24} \mathrm{Mg}$ & & & ${ }^{26} \mathrm{Mg} /{ }^{24}$ & & & & \\
\hline & & Min. & Mean & Max. & Min. & Mean & Max. & & & \\
\hline \multicolumn{11}{|l|}{ EK1-4-1 } \\
\hline Fassaite A & 23 & 20.0 & 21.1 & 21.6 & 36.7 & 38.9 & 39.8 & $-3.6 \pm 0.3$ & $-27.8^{\mathrm{d}}$ & $-17.9^{\mathrm{d}}$ \\
\hline Spinel A & 13 & 20.7 & 21.2 & 22.0 & 37.4 & 38.7 & 39.6 & $-3.9 \pm 0.5$ & & \\
\hline Spinel $\mathrm{B}^{\mathrm{e}}$ & 16 & 16.6 & 19.0 & 21.8 & 29.4 & 33.7 & 38.8 & $-2.9 \pm 0.8$ & $-31.9^{\mathrm{d}}$ & $-20.8^{d}$ \\
\hline \multicolumn{11}{|l|}{$\mathrm{C} 1$} \\
\hline S1 & 15 & 29.4 & 29.9 & 30.2 & 57.9 & 59.0 & 59.6 & $-1.5 \pm 0.2$ & $-11.3^{f}$ & $-1.7^{f}$ \\
\hline S1 & 15 & 28.5 & 29.4 & 30.2 & 57.1 & 57.7 & 58.5 & $-1.7 \pm 0.3$ & $-13.2^{f}$ & $-3.0^{f}$ \\
\hline$S 1 g$ & 19 & 29.3 & 30.6 & 32.1 & 57.7 & 60.4 & 63.5 & $-1.6 \pm 0.3$ & & \\
\hline$S 1^{h}$ & 14 & 28.2 & 28.3 & 30.0 & 56.3 & 57.2 & 58.4 & $-2.0 \pm 0.2$ & & \\
\hline Spinel $^{\mathbf{e}}$ & 15 & 28.6 & 29.3 & 30.5 & 54.6 & 55.9 & 59.3 & $-1.9 \pm 0.8$ & & \\
\hline Spinel & 10 & 30.7 & 32.3 & 33.9 & 61.8 & 63.9 & 67.6 & $-1.6 \pm 0.2$ & & \\
\hline \multicolumn{11}{|l|}{ B29 } \\
\hline S2 & 15 & -9.9 & -8.7 & -7.7 & -16.7 & -14.7 & -13.2 & $2.7 \pm 0.3$ & $-16.3^{i}$ & $-12.6^{\mathrm{i}}$ \\
\hline S2 & 36 & -10.5 & -9.6 & -8.7 & -18.1 & -16.7 & -14.7 & $2.6 \pm 0.2$ & & \\
\hline $\mathrm{R}^{\mathrm{j}}$ & 15 & -14.9 & -14.3 & -13.4 & -25.4 & -24.5 & -22.6 & $4.1 \pm 0.3$ & & \\
\hline NORMALS & 554 & -2.8 & $\equiv 0$ & 5.2 & -5.0 & $\equiv 0$ & 9.2 & $\equiv 0$ & & \\
\hline
\end{tabular}

${ }^{a}$ Each set consists of ten consecutive ratios. ${ }^{b}$ Raw measured ratios relative to the grand mean values ${ }^{25} \mathrm{Mg} /{ }^{24} \mathrm{Mg}=0.12475$ and ${ }^{26} \mathrm{Mg} /{ }^{24} \mathrm{Mg}=0.13569$

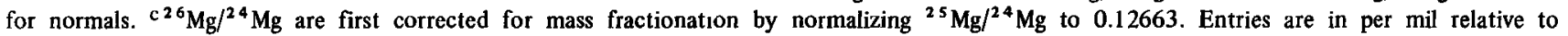
$\left({ }^{26} \mathrm{Mg} /{ }^{24} \mathrm{Mg}\right)_{0}=0.139805$. ${ }^{\mathrm{d}}$ Samples analyzed for oxygen are from a different set of mineral separates than those used for $\mathrm{Mg}$. Data from Clayton and Mayeda [1977b]. ' Analyzed using the direct loading technique [Wasserburg et al., 1977]. 'Data on splits S2 and S3 [Clayton et al., 1977]. BSecond sepa-

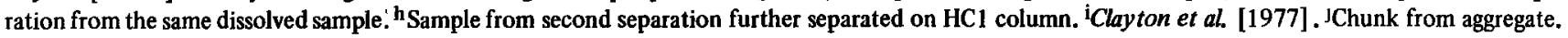

shift in the raw ratios appeared to be opposite in sign to that for $\mathrm{Cl}$, with ${ }^{25} \mathrm{Mg} /{ }^{24} \mathrm{Mg}$ and ${ }^{26} \mathrm{Mg} /{ }^{24} \mathrm{Mg} 9 \%$ and $15 \%$ respectively below the range of normals, and $\delta^{26} \mathrm{Mg}$ of $+2.5 \%$. To confirm these effects we dissolved a new fragment of B29 (B29R) and measured the $\mathrm{Mg}$ from the soluble portion. B29R showed a shift in ${ }^{25} \mathrm{Mg} /{ }^{24} \mathrm{Mg}$ and ${ }^{26} \mathrm{Mg} /{ }^{24} \mathrm{Mg}$ of $-14 \%$ and $-24 \%$ respectively relative to normal $\mathrm{Mg}$ and $\delta^{26} \mathrm{Mg}$ of $+4.1 \pm$ $0.3 \%$. The data again appear to indicate significant $\mathrm{Mg}$ isotopic shifts governed by fractionation. However, an aliquot from B29 sent to R. N. Clayton was found to lie essentially on the NE oxygen mixing line [Clayton et al., 1977].

\section{Discussion}

The data presented here show that the constituent phases in two $\mathrm{Ca}-\mathrm{Al}$ rich inclusions from Allende have $\mathrm{Mg}$ isotopic abundance patterns which: 1) appear to be highly fractionated and enriched in the heavier isotopes; 2) when normalized, yield negative $\delta^{26} \mathrm{Mg}$; and 3 ) have $O$ compositions which are displaced from the NE oxygen line. A third inclusion: 1) has highly fractionated $\mathrm{Mg}$ but is depleted in the heavier isotopes; 2) when normalized, gives positive $\delta^{26} \mathrm{Mg}$; and 3) $\mathrm{O}$ is not displaced from the NE line.

As this class of isotopic anomalies appears to suggest both Fractionation and Unidentified Nuclear effects, we will designate them FUN anomalies. The first basic question regarding the FUN anomalies is whether the $\mathrm{Mg}$ isotopic patterns which trend along a simple fractionation line shown in

Table 2. Quantitative Electron Microprobe Analysis of EK1-41 ${ }^{\mathrm{a}}$

\begin{tabular}{ccrrrr}
\hline \multicolumn{2}{c}{ Sample A \& B, Spinel } & & \multicolumn{3}{c}{ Sample B, Pyroxene } \\
\cline { 2 - 4 } \cline { 4 - 6 } $\mathrm{SiO}_{2}$ & 0.2 & & 40.3 & 42.0 & 41.3 \\
$\mathrm{TiO}_{2}$ & 0.4 & & $<0.1$ & 1.6 & 6.6 \\
$\mathrm{Al}_{2} \mathrm{O}_{3}$ & 71.9 & & 25.0 & 21.1 & 17.5 \\
$\mathrm{Cr}_{2} \mathrm{O}_{3}$ & 0.8 & & 0.3 & 0.2 & 0.2 \\
$\mathrm{FeO}$ & 0.1 & & $<0.1$ & $<0.1$ & $<0.1$ \\
$\mathrm{MnO}$ & $<0.1$ & & 0.1 & 0.1 & 0.2 \\
$\mathrm{MgO}$ & 27.1 & & 8.5 & 9.6 & 9.9 \\
$\mathrm{CaO}$ & $<0.1$ & & 25.7 & 24.8 & 25.5 \\
$\mathrm{Na}_{2} \mathrm{O}$ & $<0.1$ & & $<0.1$ & $<0.1$ & $<0.1$ \\
$\mathrm{SUM}$ & 100.5 & & 100.2 & 99.4 & 101.2 \\
\hline
\end{tabular}

${ }^{2} 2-3 \mu \mathrm{m}$ beam on $\leqslant 10 \mu \mathrm{m}$ grains. Entries in weight percent.
Fig. 2 are actually due to fractionation effects or nuclear processes. Elucidation of this matter is somewhat obfuscated by the coupling of possible fractionation effects with nonlinear effects (both positive and negative $\delta^{26} \mathrm{Mg}$ ). The good correlation of large $\delta^{26} \mathrm{Mg}$ excesses with $\mathrm{Al} / \mathrm{Mg}$ in coexisting phases in a single inclusion, in which the ${ }^{25} \mathrm{Mg} /{ }^{24} \mathrm{Mg}$ ratio is essentially normal, appears to provide a sound basis for attributing all positive $\delta^{26} \mathrm{Mg}$ values to the in situ decay of ${ }^{26} \mathrm{Al}[L P W, 1976 ; 1977]$. However, some small positive $\delta^{26} \mathrm{Mg}$ values which are attributed to ${ }^{26} \mathrm{Al}$ decay may actually be due to other nuclear processes. The demonstration of isotopic fractionation may be made most clearly when the ${ }^{25} \mathrm{Mg} /{ }^{24} \mathrm{Mg}$ and ${ }^{26} \mathrm{Mg} /{ }^{24} \mathrm{Mg}$ ratios appear to be sufficiently shifted but $\delta^{26} \mathrm{Mg}$ is small, thus indicating a mass fractionation pattern similar to eq. 1 . This is nearly the case for the three samples reported here (Fig. 2). We note that the residual shifts in $\delta^{26} \mathrm{Mg}$ are the same for different phases in the same inclusion, except that the data for B29R are displaced from B29S2. Further work will show if the $\mathrm{Mg}$ isotopic heterogeneity in B29 is correlated with $\mathrm{Al} / \mathrm{Mg}$ and "simply" due to ${ }^{26} \mathrm{Al}$ decay.

If the large shifts in $\mathbf{M g}$ abundances are due to nuclear processes, this requires special circumstances, with both production and destruction reactions such that

$\Delta 26 / /^{26} \mathrm{Mg}-\Delta 25 /{ }^{25} \mathrm{Mg} \approx \Delta 25 / /^{25} \mathrm{Mg}-\Delta 24 /{ }^{24} \mathrm{Mg}$, where $\Delta \mathrm{J}$ is the change of isotope $\mathrm{J}$ due to these reactions. Superposition of the decay of ${ }^{26} \mathrm{Al}$ and fractionation effects would account for the positive $\delta^{26} \mathrm{Mg}$ of fractionated samples if originally the $\mathrm{Mg}$ composition of this material was normal. For the other two samples, $\mathrm{Cl}$ and EK1-4-1, we must account for the negative $\delta^{26} \mathrm{Mg}$ values. This may be an artifact resulting from the procedure used to correct for fractionation There is no well-defined law for correcting for fractionation under the conditions of large fractionation effects due to multi-stage processes and subsequent mixing of fractionated reservoirs. For example, for a Rayleigh-type distillation of $\mathrm{Mg}$, both large positive $\delta^{26} \mathrm{Mg}$ and small negative $\delta^{26} \mathrm{Mg}$ can be obtained. While it is possible to produce small negative $\delta^{26} \mathrm{Mg}$ by assuming specific Rayleigh models and possibly large effects during condensation in a plasma, it is our current belief that the negative $\delta^{26} \mathrm{Mg}$ values are most likely due to nuclear effects which must result in depletion in ${ }^{24} \mathrm{Mg}$ or ${ }^{26} \mathrm{Mg}$ or in an enrichment in ${ }^{25} \mathrm{Mg}$. We cannot explain why the unknown nuclear (UN) anomalies should only occur with fractionated 


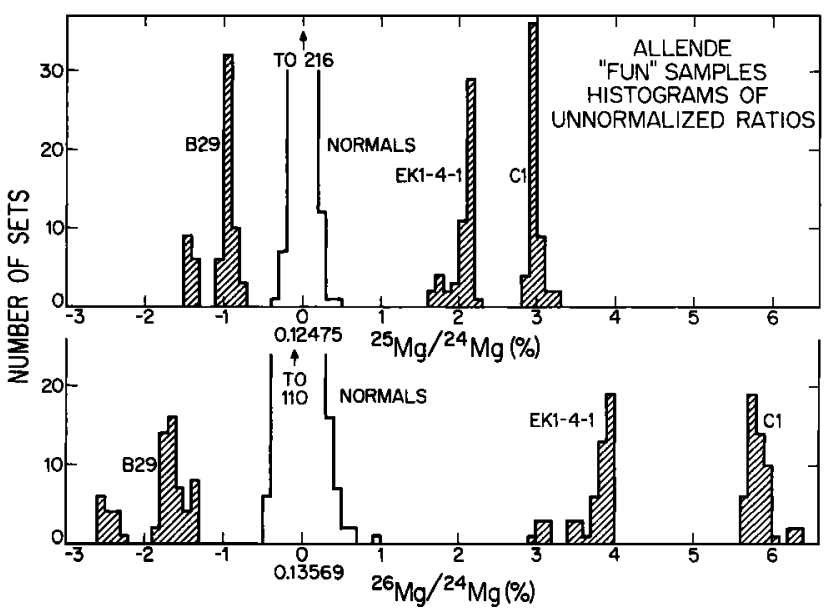

Fig. 1. Histograms of raw $\mathrm{Mg}$ isotopic ratios for FUN Ca-Al rich inclusions in Allende which contain $\mathrm{Mg}$ with highly fractionated isotopic composition ranging from $-1 \%$ to $+3 \%$ per mu, distinctly outside the instrumental fractionation range defined by the histograms of normals.

(F) Mg. As suggested by Heymann and Dziczkaniec [1976], ${ }^{26} \mathrm{Mg}$ in the solar nebula could have been depleted by bombarding the gaseous nebula with protons of energy less than $10 \mathrm{MeV}$ to produce ${ }^{26} \mathrm{Al}$ by a $(\mathrm{p}, \mathrm{n})$ reaction and at the same time removing the $\mathrm{Al}$ through rapid condensation. The bombardment of normal solar system material by $\mathrm{MeV}$ protons with fluences over $10^{20} / \mathrm{cm}^{2}$ that was needed to account for the negative effect will produce a number of other isotopic anomalies in the irradiated material. The observed effects would of course be diminished by diluting irradiated with unirradiated materials. Fowler, Greenstein and Hoyle [1962] also proposed a scenario of proton irradiation of solid objects in which further anomalies are produced by neutron capture. A recent study by Clayton, Dwek and Woosley [1977] of the proton irradiation of gas and dust has shown that the fluence necessary for ${ }^{26} \mathrm{Al}$ production will produce numerous isotopic anomalies. Other than ${ }^{26} \mathrm{Al}$, as yet no effects clearly identifiable with p-bombardment have been reported. Recent work by McCulloch and Wasserburg (pers. comm.) shows that ${ }^{135} \mathrm{Ba}$ in $\mathrm{C} 1$ appears to be depleted by $0.2 \%$ suggesting a holdup of ${ }^{135} \mathrm{Ba}$ as ${ }^{135} \mathrm{Cs}\left(\tau_{1 / 2} \sim 2 \times 10^{6}\right.$ years $)$. This points toward a possible r-process contribution.

It would appear from the available data that strong isotopic fractionation of $\mathrm{Mg}$ took place during the formation of some of the $\mathrm{Ca}-\mathrm{Al}$ chondrules in the Allende meteorite. It is important to check if Mg-poor, Al-rich phases in inclusions B29, EK 1-4-1 and $\mathrm{Cl}$ have, in addition, large positive $\delta^{26} \mathrm{Mg}$ excesses attributable to in situ ${ }^{26} \mathrm{Al}$ decay.

In order to outline a plausible mechanism for the origin of the anomalies discussed here, it is important to establish correlations between the isotopic species of different elements and in particular for those nuclides which may be genetically related by nuclear, chemical or physical processes. Two of the samples described here show a distinct departure from the NE mixing line. For Cl and EK 1-4-1 which both have enrichments in the heavy $\mathrm{Mg}$ isotopes, we note an enrichment in ${ }^{18} \mathrm{O}$ and ${ }^{17} \mathrm{O}$ relative to ${ }^{16} \mathrm{O}$ but with a smaller fractionation effect. The observed oxygen mass fractionation must reflect admixtures of extraordinary $\mathrm{O}$ and of fractionated "normal" $\mathrm{O}$; alternatively $O$ fractionation occurred after mixing of the $N$ and $E$ components. The negative $\delta^{26} \mathrm{Mg}$ appears to require extraordinary $\mathrm{Mg}$ with distinct nuclear effects. The observed $\mathrm{Mg}$ mass fractionation reflects admixture of fractionated normal and extraordinary $\mathrm{Mg}$. The simplest hypothesis would be that the $\mathrm{F}$ anomalies in $\mathrm{Mg}$ and $\mathrm{O}$ were produced simultaneously as the result of isotopic fractionation during or prior to the original formation of the chondrules. For both $\mathrm{O}$ and $\mathrm{Mg}$ the isotopic mass fractionation must have occurred prior to the crystallization of the chondrules so that the phases in a chondrule had initially a uniform isotopic composition. One conflict between $\mathrm{O}$ and $\mathrm{Mg}$ is that the $\mathrm{Mg}$ isotopic composition in $\mathrm{Cl}$ and in EK1-4-1 is uniform within each inclusion whereas the oxygen isotopic compositions of the different phases in an individual inclusion are different. Clayton and Mayeda [1977a,b] show that, in EK1-4-1, spinel and pyroxene are slightly different from each other and both are offset from the NE mixing line, while the melilite is on the mixing line much closer to "normal" oxygen. This observation for oxygen is common to many Allende inclusions [Clayton et al., 1977] and would appear to support the concept of a magical carrier phase containing the extraordinary component. However, no major variation in $\mathrm{Mg}$ between coexisting phases has been observed which cannot be explained by ${ }^{26} \mathrm{Al}$ decay, thus indicating initial $\mathrm{Mg}$ isotopic homogeneity within a single chondrule. In addition, there is a difficulty because the oxygen in B29 lies on the NE line and therefore has not been affected by the $\mathrm{Mg}$ fractionation process.

The general chemical composition of the $\mathrm{Ca}-\mathrm{Al}$ rich inclusions and chondrules in the Allende meteorite clearly suggest the approximate composition of early high temperature solar nebula condensates [Grossman, 1975]. Grossman has constructed models of sequential equilibrium condensation to explain the chemistry and mineralogy of these inclusions. There have been from the outset four major difficulties with this model: a) the presence of phases inside the chondrules which are intermediate temperature condensates; b) the abundance of alkalis and halogens cannot be part of a high temperature condensation regime; c) the morphology and type of crystal intergrowths of most chondrules indicate that they originally formed as molten droplets and subsequently crystallized; d) the artificial distinction between different types of chondrules and aggregates which have similar major element contents, although some aggregates are enriched in iron. These difficulties have been noted repeatedly in the literature [ $c f$. Blander and Fuchs, 1975]. While Allende has been subject to young metamorphism, the introduction of halogens in the inclusions cannot be related to this event because of the presence of ${ }^{129} \mathrm{Xe}$ from ${ }^{129} \mathrm{I}$ decay [Podosek and Lewis, 1972].

Blander and Fuchs [1975] have discussed the importance of metastable condensation as a process in chondrule formation and have emphasized the importance of this process for the Allende chondrules. Wark and Lovering [1977] recently demonstrated the presence on $\mathrm{Ca}-\mathrm{Al}$ rich chondrules of regular thin rims which are composed of distinct layers. These layers include $\mathrm{Fe}$-rich and $\mathrm{Na}$-rich compositions and correspond generally to materials condensing at relatively lower temperatures than the interior $\mathrm{Ca}-\mathrm{Al}$ rich materials. These observations appear to indicate that both non-equilibrium condensation directly to metastable, subcooled liquids, and direct equilibrium condensation from gas to solid must be invoked for the

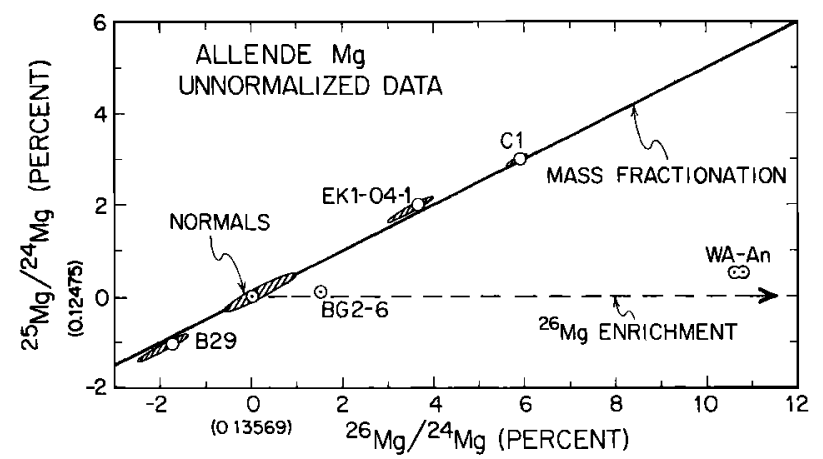

Fig. 2. Plot of fractional variations of raw ${ }^{25} \mathrm{Mg} /{ }^{24} \mathrm{Mg}$ versus ${ }^{26} \mathrm{Mg} /{ }^{24} \mathrm{Mg}$. The data are close to the slope $1 / 2$ line indicating significant mass fractionation effects. Inclusions $\mathrm{Cl}$ and EK1-4-1 show distinct deviations from this line corresponding to negative $\delta^{26} \mathrm{Mg}$. 
production of $\mathrm{Ca}-\mathrm{Al}$ rich chondrules. Wark and Lovering [1977] also established that the $\mathrm{Ca}-\mathrm{Al}$ rich aggregates are composed of loosely compacted microscopic, zoned bodies whose composition corresponds to the compositions of the rims of the $\mathrm{Ca}-\mathrm{Al}$ rich chondrules. We note that we have obtained identical initial ${ }^{26} \mathrm{Al} /{ }^{27} \mathrm{Al}$ for two chondrules in Allende [ $L P W 1976,1977]$. One of these chondrules (WA) is a typical Ca-Al rich chondrule containing melilite, spinel, pyroxene and anorthite, whereas the other (BG2-6) is composed of hedenbergite and grossular and is similar in composition to the rims observed by Wark and Lovering [1977]. The ${ }^{26} \mathrm{Al} /{ }^{27} \mathrm{Al}$ requires that the higher temperature chondrules and the Fe-rich rims and aggregates condensed within less than $10^{5}$ years.

For the interpretation of the $\mathrm{O}$ and $\mathrm{Mg}$ data we adopt the general point of view of Blander and Fuchs [1975] and hypothesize that metastable (supercooled) liquid droplets were present in the early solar nebula which contained those elements associated with general equilibrium condensation over a wide and somewhat variable temperature regime $\left(1700-1200^{\circ} \mathrm{K}\right)$. This permits subsequent zoning by crystallization, and the presence of late high-temperature phases. We further assume that these chondrules were originally isotopically homogeneous in their constituent phases and with an $O$ composition highly anomalous with $\delta^{17} \mathrm{O} \sim \delta^{18} \mathrm{O} \leqslant-40 \%$. The $\mathrm{O}$ and $\mathrm{Mg}$ anomalies are then characteristic of the original bulk chondrule, and not due to the admixture of a carrier phase or of interstellar dust grains. After crystallization of the chondrules, the rim materials condensed at slightly lower temperature, and the chondrules were then immersed in a region of the solar nebula which was at a relatively low temperature and contained $O$ of nearly normal composition. This happened on a short time scale and caused the major alteration of the chondrules and aggregates. At this time the alkalis and halogens including ${ }^{129} \mathrm{I}$ were selectively impregnated into the inclusions. During this back-reaction with the cool solar nebula there was a significant exchange of oxygen between those phases which were most reactive, particularly plagioclase and melilite, and the lower temperature materials in the aggregates. The only major problem is the difficulty of such extensive $O$ exchange in a low density medium. Using this model, we attempt to explain the FUN anomalies. The displacement of the oxygen data from the mixing line for the $C 1$ and EK1-4-1 inclusions is due to isotopic fractionation in the material from which the chondrules formed. This fractionation is manifest in the $\mathrm{Mg}$ data for all phases because there is too little $\mathrm{Mg}$ remaining in the cool solar nebula to cause any significant shift during back-reaction. The spinel and pyroxene in EK 1-4-1 reflect this isotopic fractionation for oxygen, but the pyroxene is slightly altered by back-reaction. The melilite back-reacted to a large extent so that its oxygen value shifted close to that for the "normal" solar system value. This model implies that the oxygen isotopic data on other Ca-Al chondrules not of the FUN type also reflect the same general process and that the reason for $O$ isotopic heterogeneities between mineral phases in a single inclusion is the result of differential response of various minerals during back-reaction with the solar nebula rather than a reflection of original isotopic heterogeneities within the chondrules. The lower limit of $\delta^{18} \mathrm{O} \sim-40 \%$ [Clayton et al., 1977] is interpreted as the starting oxygen composition of these inclusions before alteration. In this model, the amount of normal oxygen added to this starting oxygen composition during back-reaction correlates with the amount of lowtemperature material which was added at the same time. Thus the oxygen shifts should show an anti-correlation with the alkali and halogen contents for the inclusions. This model does not explain where the original $O$ anomaly or the negative $\delta^{26} \mathrm{Mg}$ came from and still requires an isotopically heterogeneous solar nebula. The lack of correlation for B29 remains a problem. This interpretation of distinct $O$ isotopic compositions for different phases within single inclusions was proposed first by Blander and Fuchs [1975].
The nuclear, physical and chemical processes which generated the exotic $\mathrm{O}$, the ${ }^{26} \mathrm{Al}$ and the other non-linear $\mathrm{Mg}$ isotopic anomalies as well as the observed isotope fractionation are not addressed by this model. The distinct shifts of oxygen between the many classes of meteorites show that the contribution of exotic nuclei to the whole solar system was widespread and significant and the ${ }^{26} \mathrm{Al}$ indicates that the time scale between production and condensation was very short unless ${ }^{26} \mathrm{Al}$ was produced with concurrent condensation over a long time.

Acknowledgements. We thank $\mathrm{H}$. Nagasawa for generously providing us materials from inclusion EK1-4-1. We thank D. Heymann for coming out of the trench where he was hiding from pre-solar grain dum-dums and supernovatic grenades to review this paper. This work was supported by NSF grant PHY 76-02724 and NASA grant NGL 05-002-188.

\section{References}

Blander, M., and L.H. Fuchs, Ca-Al-rich inclusions in Allende: evidence for liquid origin, Geochim. Cosmochim. Acta, 39, 1605-1619, 1975.

Catanzaro, E.J., and T.J. Murphy, Magnesium isotope ratios in natural samples, J. Geophys. Res., 71, 1271,1966.

Clayton, D.D., E. Dwek, and S.E. Woosley, Isotopic anomalies and proton irradiation in the early solar system, $A p . J ., 1977$ (submitted).

Clayton, R.N., L. Grossman, and T.K. Mayeda, A component of primitive nuclear composition in carbonaceous meteorites, Science, 182, 485-488, 1973.

Clayton, R.N., and T.K. Mayeda, Anomalous anomalies in carbonaceous chondrites, Lunar Science VIII (The Lunar Sci. Inst.) 193-195, 1977a.

Clayton, R.N., and T.K. Mayeda, Correlated oxygen and magnesium isotope anomalies in Allende inclusions: I. Oxygen, Geophys. Res. Lett., 4, $1977 \mathrm{~b}$.

Clayton, R.N., N. Onuma, L. Grossman, and T.K. Mayeda, Distribution of the pre-solar component in Allende and other carbonaceous chondrites, Earth Planet. Sci. Letters, 34, 209-224, 1977

Fowler, W.A., J.L. Greenstein, and F. Hoyle, Nucleosynthesis during the early history of the solar system, Geophys. J. Roy. Astron. Soc., 6, 148-220, 1962.

Gray, C.M., and W. Compston, Excess ${ }^{26} \mathrm{Mg}$ in the Allende meteorite, Nature, 251, 495-497, 1974.

Gray, C.M., D.A. Papanastassiou, and G.J. Wasserburg, The identification of early condensates from the solar nebula, Icarus, 20, 213-239, 1973

Grossman, L., Condensation in the primitive solar nebula, Geochim. Cosmochim. Acta, 36, 597-618, 1972.

Grossman, L., Petrography and chemistry of Ca-rich inclusions in the Allende meteorite, Geochim. Cosmochim. Acta, 39, 433-454, 1975.

Heymann, D., and M. Dziczkaniec, Early irradiation of matter in the solar system: magnesium (p,n) scheme, Science, 191, 79.81, 1976.

Lee, T., and D.A. Papanastassiou, Mg isotopic anomalies and correlation with $\mathrm{O}$ and $\mathrm{Sr}$ effects, Geophys. Res. Letters, 1, 225-228, 1974.

Lee, T., D.A. Papanastassiou, and G.J. Wasserburg, Demonstration of ${ }^{26} \mathrm{Mg}$ excess in Allende and evidence for ${ }^{26} \mathrm{Al}$, Geophys. Res. Letters, 3, 109-112, 1976.

Lee, T., D.A. Papanastassiou, and G.J. Wasserburg, Aluminum-26 in the early solar system' fossil or fuel?, Ap. J., 211, L107-L110, 1977.

Lord, H.C., III, Molecular equilibria and condensation in a solar nebula and cool stellar atmospheres, Icarus, 4, 279-288, 1965.

Podosek, F.A., and R.S. Lewis, ${ }^{129} \mathrm{I}$ and ${ }^{244} \mathrm{Pu}$ abundances in the Allende meteorite, Earth Planet. Sct. Letters, 15, 101-109, 1972.

Schramm, D.N., F. Tera and G.J. Wasserburg, The isotopic abundance of ${ }^{26} \mathrm{Mg}$ and limits on ${ }^{26} \mathrm{Al}$ in the early solar system, Earth Planet. Scl Letters, 10,44-59, 1970.

Wark, D.A., and J.F. Lovering, Marker events in the early evolution of the solar system: evidence from rims on Ca-Al-rich inclusions in carbonaceous chondrites, in Lunar Science VIII (The Lunar Sci. Inst.) 976-978, 1977.

Wasserburg, G.J., T. Lee, and D.A. Papanastassiou, $\mathrm{Mg}$ and $\mathrm{Ca}$ isotopic study of individual microscopic crystals from the Allende meteorite by the direct loading technique, in Lunar Science VIII (The Lunar Sci. Inst.) 991-993, 1977.

(Received April 15, 1977; accepted Apri1 28, 1977.) 